\title{
Correction to: Gleason-Type Theorems from Cauchy's Functional Equation
}

\author{
Victoria J. Wright ${ }^{1}$. $\cdot$ Stefan Weigert ${ }^{1}[$
}

Published online: 15 April 2020

(c) Springer Science+Business Media, LLC, part of Springer Nature 2020

\section{Correction to: Foundations of Physics (2019) 49:594-606 https://doi.org/10.1007/s10701-019-00275-x}

The authors would like to make the corrections to the original article described below.

1. Page 594-597, 604: Replace "additive" with "finitely additive" throughout the paper.

2. Page 595: Replace the first line "Gleason concluded that the solutions of Eq. (1) necessarily admit an expression" with "Gleason's result shows that the solutions of Eq. (1) (in finite dimensional Hilbert spaces) $)^{1}$ necessarily admit an expression", including Footnote 1.

3. Page 595: Replace the line preceeding Eq. (3) "Then, any function $f: \mathcal{E}(\mathcal{H}) \rightarrow[0,1]$ satisfying additivity ..." with "Then, in finite dimensional Hilbert spaces, any function $f: \mathcal{E}(\mathcal{H}) \rightarrow[0,1]$ satisfying finite additivity ...". 4. Page 596-597: Replace the proof of Theorem 1:

\footnotetext{
${ }^{1}$ Gleason proved this result in all separable Hilbert spaces if the condition of finite additivity is replaced with $\sigma$-additivity. These conditions are equivalent in finite dimensional Hilbert spaces. Christensen [1] showed that the weaker condition of finite additivity is also sufficient for the result to hold in infinite dimensions.
}

The original article can be found online at https://doi.org/10.1007/s10701-019-00275-x.

Victoria J. Wright vw550@york.ac.uk

Stefan Weigert

stefan.weigert@york.ac.uk

1 Department of Mathematics, University of York, York YO10 5DD, UK 
Theorem 1 Let $a>0$ and $f:[0, a] \rightarrow \mathbb{R}$ be a function that satisfies

$$
f(x)+f(y)=f(x+y),
$$

for all $x, y \in[0, a]$ such that $(x+y) \in[0, a]$. The function $f$ is necessarily linear, i.e.

$$
f(x)=\frac{f(a)}{a} x,
$$

if it satisfies any one of the following four conditions:

(i) $f(x) \leq b$ for some $b \geq 0$ and all $x \in[0, a]$;

(ii) $f(x) \geq c$ for some $c \leq 0$ and all $x \in[0, a]$;

(iii) $f$ is continuous at zero;

(iv) $f$ is Lebesgue-measurable.

Theorem 1 says that non-linear solutions of Eq. (1) cannot be bounded from below or above, continuous at zero or Lebesgue measurable. We will now prove the linearity of $f$ for Case (i). The proofs for the remaining cases are given in Appendix A.

Proof We will extend $f$ to a finitely additive function on the entire real line. For any real number $x \in[0, a]$, Eq. (1) implies that

$$
f(x)=f\left(\frac{n}{n} x\right)=n f\left(\frac{x}{n}\right),
$$

where $n$ is a positive integer. If we choose an integer $m \in \mathbb{N}$ with $m / n \in[0, a]$, then we have

$$
f\left(\frac{m}{n} x\right)=m f\left(\frac{x}{n}\right)=\frac{m}{n} f(x) .
$$

In a first step, we extend the function $f$ to all non-negative real numbers by defining

$$
f_{+}(x)=n f\left(\frac{x}{n}\right)
$$

for real numbers $x>a$ and integers $n>x / a$. This extension is well-defined since for any two sufficiently large integers, i.e. $m$ and $n$ with $m, n>x / a$, we have

$$
f\left(\frac{x}{m n}\right)=\frac{1}{m} f\left(\frac{x}{n}\right)=\frac{1}{n} f\left(\frac{x}{m}\right)
$$

according to Eq. (3), resulting in the identity

$$
m f\left(\frac{x}{m}\right)=n f\left(\frac{x}{n}\right) .
$$


Finite additivity on the positive half-line also holds since for any two non-negative numbers $x, y \geq 0$, we find

$$
\begin{aligned}
f_{+}(x)+f_{+}(y) & =n f\left(\frac{x}{n}\right)+n f\left(\frac{y}{n}\right) \\
& =n f\left(\frac{x+y}{n}\right)=f_{+}(x+y),
\end{aligned}
$$

for sufficiently large $n \in \mathbb{N}$ which ensures that $(x+y) / n \in[0, a]$.

In a second step, we extend the function $f_{+}$to the entire real line by defining

$$
f_{\mathbb{R}}= \begin{cases}+f_{+}(x), & \text { for } x \geq 0, \\ -f_{+}(x), & \text { for } x<0 .\end{cases}
$$

To show that the function $f_{\mathbb{R}}$ is finitely additive on all of $\mathbb{R}$, three cases must be considered.

If both $x<0$ and $y<0$, we have

$$
\begin{aligned}
f_{\mathbb{R}}(x)+f_{\mathbb{R}}(y) & =-f_{+}(-x)-f_{+}(-y) \\
& =-f_{+}(-x-y)=f_{\mathbb{R}}(x+y),
\end{aligned}
$$

using that $f_{+}(-x)+f_{+}(-y)=f_{+}(-x-y)$ holds for non-negative real numbers $-x$ and $-y$.

If $x \geq 0, y<0$ and $x+y<0$, we have

$$
\begin{aligned}
f_{\mathbb{R}}(x)+f_{\mathbb{R}}(y) & =f_{+}(x)-f_{+}(-y-x+x) \\
& =f_{+}(x)-f_{+}(-y-x)-f_{+}(x)=f_{\mathbb{R}}(x+y) .
\end{aligned}
$$

If $x \geq 0, y<0$ and $x+y \geq 0$, we have

$$
\begin{aligned}
f_{\mathbb{R}}(x)+f_{\mathbb{R}}(y) & =f_{+}(x+y-y)-f_{+}(-y) \\
& =f_{+}(x+y)+f_{+}(-y)-f_{+}(-y)=f_{\mathbb{R}}(x+y) .
\end{aligned}
$$

This property completes the proposed extension of the function $f$ to a finitely additive function $f_{\mathbb{R}}$ on the real line that is bounded from above on the interval $[0, a]$. Ostrowski [2] and Kestelman [3] showed that finitely additive functions on the real line that are bounded from above on a set of positive measure are necessarily linear. Therefore, the extended function $f_{\mathbb{R}}$ is linear, and its restriction back to the interval $[0, a]$ is given by $f(x)=f(a) x / a$.

5. Page 604: Add to the acknowledgements "The authors thank Jonathan Barrett for pointing out a gap in the proof of Theorem 1 given in the published paper."

6. Page 606: New references added: $[1,2]$. 


\section{References}

1. Christensen, E.: Measures on projections and physical states. Commun. Math. Phys. 86(4), 529 (1982)

2. Ostrowski, A.: Über die Funktionalgleichung der Exponentialfunktion und verwandte Funktionalgleichungen. Jahresber. Dtsch. Math. Verh. 38, 54 (1929)

3. Kestelman, H.: On the functional equation $f(x+y)=f(x)+f(y)$. Fundam. Math. 1(34), 144 (1947)

Publisher's Note Springer Nature remains neutral with regard to jurisdictional claims in published maps and institutional affiliations. 